\title{
Calcination of Pd Nanoparticles on Delta Alumina : Ex-situ Analysis versus In-situ Environmental TEM
}

Siddardha Koneti ${ }^{1}$, Lucian Roiban ${ }^{1}$, Anne-Sophie Gay $^{2}$, Priscilla Avenier ${ }^{2}$, Florent Dalmas ${ }^{1}$ and Thierry Epicier ${ }^{1}$

1. MATEIS, UMR 5510, Université de Lyon, CNRS, INSA de Lyon, UCB Lyon 1, 69621 Villeurbanne Cedex, France.

2. IFP Energies nouvelles -Rond-point de l'échangeur de Solaize - BP 3, 69360 Solaize - France.

Sustainable chemistry, reduction of pollution, greenhouse gases control, oil refinery, liquid and solid waste management, are essential societal topics related to what is called Environmental catalysis [1], a sector which is booming rapidly for the past 10 years. So, developing innovative catalysts is a very important aspect ahead of us. To develop more advanced catalysts, we have to understand the catalysts genesis in all the stages of preparation (impregnation, drying, calcination and reduction of the active phase). Nowadays Environmental Transmission Electron Microscopy (ETEM) enables dynamic studies of the catalytic activity down to the nanometer and even atomic scale.

This contribution shows the dynamic evolution of palladium nanoparticles (NP) supported by alumina during the catalysis preparation process. Here, the calcination step is studied in a FEI TITAN-ETEM microscope operated at $300 \mathrm{kV}$. A Pd/delta alumina catalyst was investigated at different temperatures and under different gas pressures in order to follow the particles size evolution. Owing to the small size of the Pd NPs in the range of 1-3 nm, we mainly used the STEM-ADF imaging mode. we performed in-situ in order to ascertain the chemical nature of the observed particles. During this work it appeared essential perform measurements systematically on the same areas at different temperatures. Fig. 1 is a typical illustration of the in-situ evolution of Palladium particles under Oxygen partial pressure at different temperatures. From such micrographs, the NP size evolution was quantified and compared with post-mortem TEM observations after ex-situ experiments performed at same temperatures but atmospheric pressure in the course of the catalyst synthesis (Fig. 2). This comparison shows that all measurements appear to be consistent except those performed in the bright field TEM imaging mode, where larger particles sizes are obtained, most probably due to irradiation effects which were further evidenced by a high-induced mobility of NPs on their supporting media. This environmental study brings direct in situ information on the transient stages that cannot be followed by post mortem experiments after ex-situ treatment [2].

\section{References:}

[1] PG Centi et al, Catalysis Today 75 (2002), pp. 3-15.

[2] The CLYM (Consortium Lyon - St-Etienne de Microscopie, www.clym.fr) is acknowledged for its guidance in the ETEM project which was financially supported by the CNRS, the Région Rhône-Alpes, the 'GrandLyon' and the French Ministry of Research and Higher Education. 

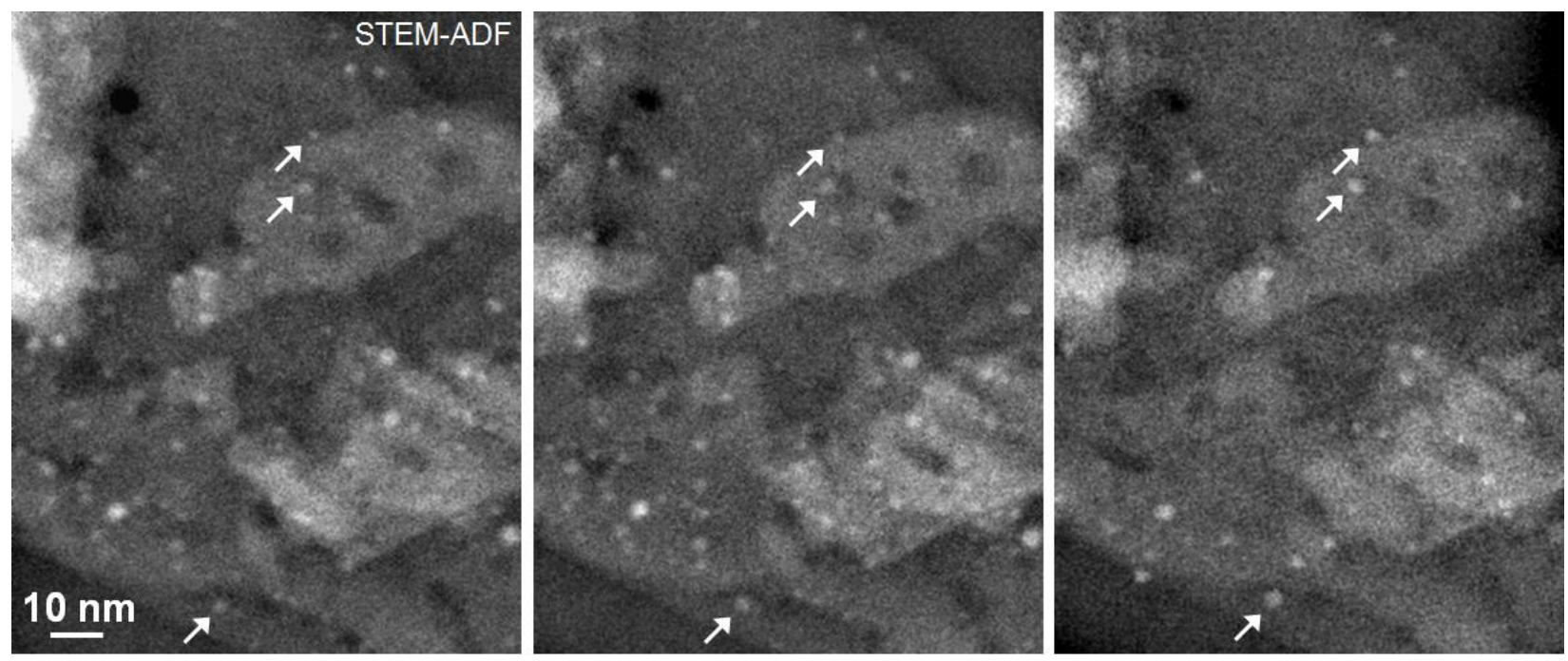

Figure 1. Evolution of Pd NPs on delta alumina support: STEM-ADF imaging in ETEM under 0.2 mbar of oxygen at temperature from 20 to 150 and $425^{\circ} \mathrm{C}$ respectively from left to right. Coalescence occurs as attested by the disappearance of some small particles and the growth of others (such as pointed by arrows).

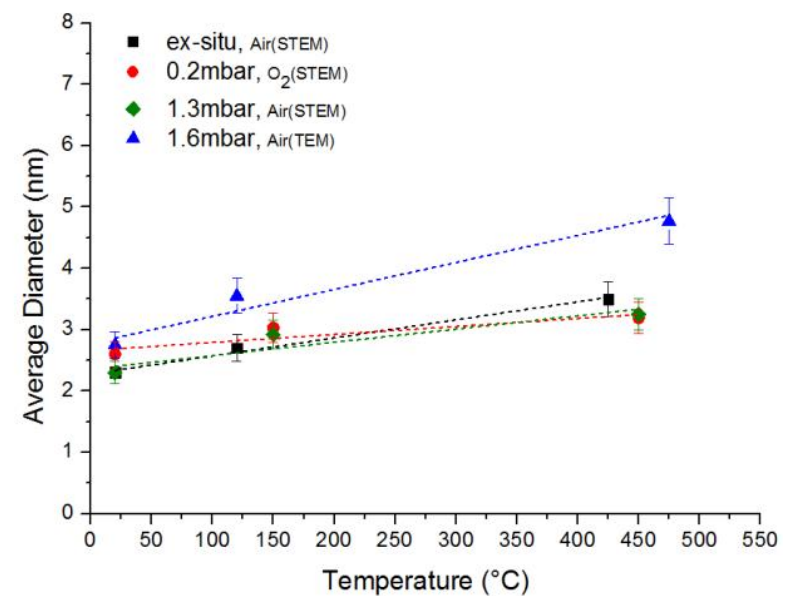

Figure 2. Size evolution of Pd particles at different pressures compared whit the post mortem analysis of ex-situ experiment. 